\title{
Embedding silica and polymer fibre Bragg gratings (FBG) in plastic 3D-printed sensing patches
}

\author{
Michal G. Zubel ${ }^{a^{*}}$, Kate Sugden ${ }^{\mathrm{a}}$, David J Webb ${ }^{\mathrm{a}}$, \\ David Saez-Rodriguez ${ }^{\mathrm{b}}$, Kristian Nielsen ${ }^{\mathrm{c}}$, Ole Bang ${ }^{\mathrm{c}}$ \\ ${ }^{a}$ Aston Institute of Photonic Technologies, Aston University, B4 7ET Birmingham, United Kingdom \\ ${ }^{\mathrm{b}}$ Optical and Quantum Communications Group, ITEAM Research Institute, Universitat Politecnica \\ de Valencia, 46022 Valencia, Spain \\ ${ }^{c}$ DTU Fotonik, Department of Photonics Engineering, DK-2800 Kgs. Lyngby, Denmark
}

\begin{abstract}
This paper reports the first demonstration of a silica fibre Bragg grating (SOFBG) embedded in an FDM 3-D printed housing to yield a dual grating temperature-compensated strain sensor. We also report the first ever integration of polymer fibre Bragg grating (POFBG) within a 3-D printed sensing patch for strain or temperature sensing. The cyclic strain performance and temperature characteristics of both devices are examined and discussed.

The strain sensitivities of the sensing patches were 0.40 and $0.95 \mathrm{pm} / \mu \varepsilon$ for $\mathrm{SOFBG}$ embedded in ABS, $0.38 \mathrm{pm} / \mu \varepsilon$ for POFBG in PLA, and $0.15 \mathrm{pm} / \mu \varepsilon$ for POFBG in ABS. The strain response was linear above a threshold and repeatable. The temperature sensitivity of the SOFBG sensing patch was found to be up to $169 \mathrm{pm} /{ }^{\circ} \mathrm{C}$, which was up to 17 times higher than for an unembedded silica grating. Unstable temperature response POFBG embedded in PLA was reported, with temperature sensitivity values varying between 30 and $40 \mathrm{pm} /{ }^{\circ} \mathrm{C}$.
\end{abstract}

Keywords: polymer optical fibre, fibre Bragg grating, FBG, POFBG, additive layer manufacturing, 3-D printing, strain sensor, temperature sensor

\section{INTRODUCTION}

3D printing techniques (also known as additive layer manufacturing, ALM) have in recent years been revolutionising many fields of engineering ${ }^{1,2}$, owing to their relatively low price and hence wide accessibility, ease of producing random shapes and a growing number of available materials. Fused deposition modelling (FDM) technique, being the most popular ALM approach, has certain advantages from the viewpoint of FBG embedding, which include availability of printing materials of various properties (different flexibility, chemical composition, thermal expansion coefficient etc.), ability to mix different materials within one 3D-printed structure, as well as ease of fabrication of empty cavities, channels, beams, membranes and other types of functional elements.

Polymer optical fibre Bragg gratings (POFBGs) are an emerging yet promising type of optical fibre sensors, which possess important advantages over its silica counterparts (SOFBGs). These include a smaller Young's modulus (3.3 GPa for a PMMA fibre compared to $73 \mathrm{GPa}$ for a silica one) and thus higher flexibility, ease of modification of chemical composition, which influences the properties of fibre (e.g. affinity to water or lack thereof), biocompatibility and biodegradability ${ }^{3}$. These unique properties of POFBGs gave rise to new applications in various fields, even as distant as e.g. medicine $e^{4,5}$ and aviation industry ${ }^{6}$.

This study focuses on embedding both SOFBGs and POFBGs in sensing patches produced using FDM. Silica FBGs have previously been embedded in structures produced by means of $\mathrm{ALM}^{7}$, including those integrated with FDM-fabricated structures made of acrylonitrile butadiene styrene $(\mathrm{ABS})^{8}$, but the latter was not used for sensor fabrication. In addition, embedding POFBGs in 3D-printed structures is reported in this paper for the first time. The authors managed to successfully embed POFGBs in ABS and polylactic acid (PLA), which are the most widespread FDM filament materials. Combining assets of both POFBGs and ALM is seen as an important step towards making POFBGs more widely applicable and more easily customisable, hence letting them find their way to the market.

*m.zubel@aston.ac.uk

Micro-Structured and Specialty Optical Fibres IV, edited by Kyriacos Kalli,

Alexis Mendez, Proc. of SPIE Vol. 9886, 98860N · (c) 2016 SPIE

CCC code: $0277-786 \mathrm{X} / 16 / \$ 18 \cdot$ doi: $10.1117 / 12.2228753$

Proc. of SPIE Vol. 988698860 N-1 


\section{FBG INSCRIPTION}

Both the SOFBGs and POFBGs were inscribed in-house by scanning a UV beam across a phase mask ${ }^{9}$ using a Kimmon $325 \mathrm{~nm} \mathrm{HeCd}$ laser (35 mW, model IK3301 R-G). The experimental set-ups used during the inscription are shown in Figure 1.

\subsection{SOFBGs}

Two gratings spaced by $50 \mathrm{~mm}$ (centre to centre) were inscribed through-coating on one piece of hydrogen-loaded Fibercore PS 1250/1500 fibre using an Ibsen Photonics custom-made phase mask (1061.18 nm pitch). The scanning length, speed and time of both gratings were $9 \mathrm{~mm}, 7.4 \mu \mathrm{m} / \mathrm{s}$ and $12 \mathrm{~min}$, respectively. The resulting gratings had the Bragg peak wavelength and power of $1536.7 \mathrm{~nm}$ and $-47.8 \mathrm{dBm}$ (SOFBG1), and $1530.4 \mathrm{~nm}$ and $-50.0 \mathrm{dBm}$ (SOFBG2) respectively. SOFBG2 was strained before inscription, in order to give a Bragg wavelength difference between the two gratings.

\subsection{POFBGs}

Two POFBGs were inscribed using an Ibsen Photonics custom-made phase mask (557.50 nm pitch) on a highlyphotosensitive $125 \mu \mathrm{m}$ microstructured POF made of poly(methyl methacrylate) (PMMA) doped with benzyl dimethyl ketal $(\mathrm{BDK})^{10}$. The scanning length, speed and time of the grating embedded in PLA (PLA-POFBG) and ABS (ABSPOFBG) were both $5.2 \mathrm{~mm}, 2.2 \mu \mathrm{m} / \mathrm{s}$ and $30 \mathrm{~min}$, respectively. The resulting gratings had Bragg peak wavelength and power of $828.3 \mathrm{~nm}$ and $-68.4 \mathrm{dBm}$ (PLA-POFBG), and $828.4 \mathrm{~nm}$ and $-68.2 \mathrm{dBm}$ (ABS-POFBG) respectively.

a)

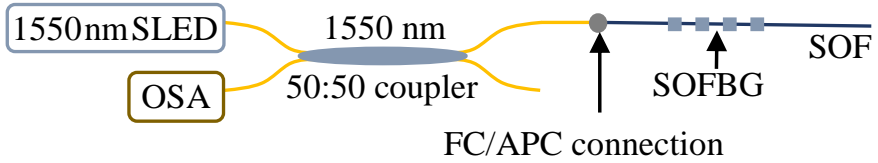

b)

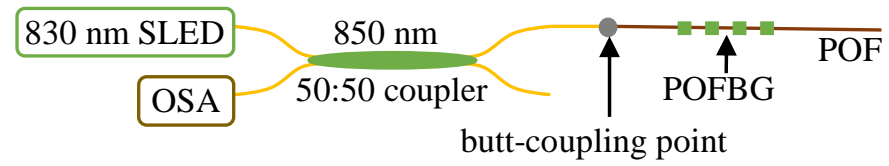

Figure 1. Experimental setup for a) SOFBG inscription, and b) for POFBG inscription and interrogation.

\section{FBG EMBEDDING}

All sensing patches housing the FBGs were fabricated with an UP! printer (model 3DP-14-4A).

The embedding procedure was similar for both SOFBGs and POFBGs, the differences are given in the respective subsections. The 3-D printing process was paused midway, at the height at which the full depth of the embedding channel was reproduced, and just before deposition of the layer that was about to cover it. In cases when glue was used, it was then placed at the bottom of the channel, before fibre was inserted. The presence and type of glue, as well as deposition procedure varied depending on the sample. Finally, the printing process was resumed to complete the housing structure.

3 -D designs of sensing patches with the grating embedding points marked on them are shown in Figure 2.

\subsection{SOFBGs}

The housing structure prepared for the silica gratings was intended to demonstrate the possibility of embedding multiple gratings in one sensing patch, each one of which can be set to sense different physical quantities. In this particular case, two gratings embedded in one patch were meant to provide temperature-insensitive strain reading. Strain was applied between the fixing points on the two mounting supports perpendicular to the channel, one of which was clamped to a translation stage. SOFBG1 (or temperature-sensing grating, T-FBG) was placed outside the straining region, hence was expected not to sense any strain. SOFBG2 (or strain-sensing grating, $\varepsilon$-FBG) was sensitive to both strain and temperature. Interrogating both gratings at the same time is the easiest solution to one of the classical problems of FBG cross-sensitivity to temperature, which always occurs on sensing other physical quantities. 
a)

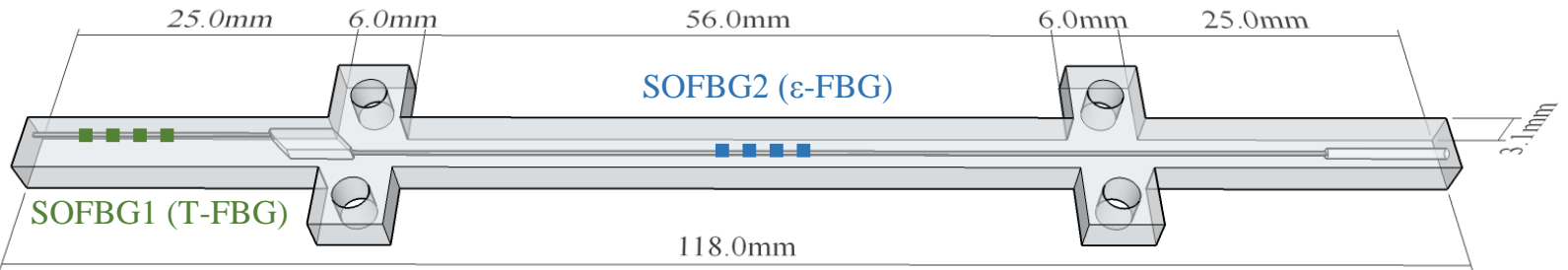

b)

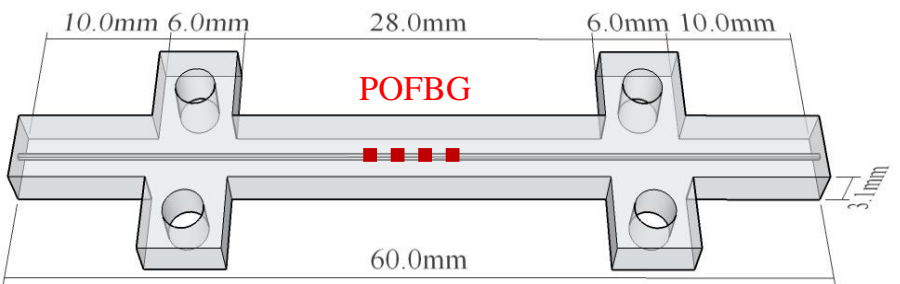

Figure 2. 3-D view of the housing structures for a) SOFBGs and b) PLA-POFBG (the design for ABS-POFBG is almost the same as $b$, see text for details). Dotted line marks the position in which gratings are embedded.

The structural material used to construct the patch was ABS, as it has better mechanical properties than PLA, another popular filament type used in FDM. The depth of the embedding channel was set to $150 \mu \mathrm{m}$, which is the smallest reproducible dimension with the 3D printer used (1 layer thickness). For the sake of robustness, secondarily coated silica fibre was directly integrated into the sensing patch (see the rightmost part of the structure in Figure 2a).

The final design of SOFBG housing patch resulted from multiple design tests and embedding trails that were performed in order to optimise the structure (Figure 2a). There were two main aspects of investigation:

- $\quad$ appropriate housing structure design, ensuring that the T-FBG was not cross-sensitive to strain

- $\quad$ search for appropriate adhesion strength between fibre and structure

The former problem was noticed, when both gratings were placed in one continuous channel (as in Figure 2b). The T-FBG was still able to detect about $1 / 3$ of strain magnitude sensed by the $\varepsilon-\mathrm{FBG}$, despite being placed outside the straining region (limited by the perpendicular supports). This was believed to be due to high silica fibre stiffness compared to the sensing patch material, which resulted in strain transfer outside the straining region.

A good solution to that came from one of the distinctive characteristics of 3D-printing techniques, namely ability to easily incorporate empty cavities in printed designs. An air cavity was placed between the two gratings, and the channel for the T-FBG was offset from the central position. These resulted in a fibre slack, ensuring that any strain transferred by the fibre from the straining region relaxed in the empty cavity. This approach prove to be successful, yielding no strain induced in the straining region being sensed by the T-FBG.

The other main issue related to silica fibre embedding, namely the need for optimising adhesion strength between fibre and housing structure, became evident when unfavourable fibre behaviour in the early print-outs was noticed. It consisted of apparent hysteresis on quick bending or straining of the structures by hand, which resulted in "zero level shifting" (random changes of Bragg peak wavelength for a relaxed structure after bending or straining it). Another symptom of insufficient fibre-structure adhesion was Bragg peak quickly moving back towards initial wavelength after straining or bending of the structure. Finally, on temperature increase, when structure was expanding, abrupt jumps related to fibre relaxing accumulated stress were noticed. These effects were believed to be associated with fibre sliding against the housing structure, coming from insufficient fibre-structure adhesion strength.

The effects enumerated above were visible with smaller or higher magnitude for all of the following embedding approaches:

- $\quad$ fibre with uncoated SOFBG region embedded directly into the structure without using glue 
- fibre with uncoated SOFBG region embedded using ethyl 2-cyanoacrylate + poly(methacrylate-co-ethyl acrylate) (commonly known as Super Glue), deposited on the entire fibre length

- the same as above, but leaving the SOFBG region free from glue

- fibre with uncoated SOFBG region glued with (3-(trimethoxysilyl)propyl)ethylene-diamine, deposited on the entire fibre length; it is a popular elastic glue, which initially bonds in $5 \mathrm{~min}$, and fully cures in 1 hour.

As none of the tested embedding approaches prove to yield satisfying results from the viewpoint of sensor applications, through coating inscribed SOFBGs bonded to the patch using Super Glue were tested in the next step. Their inscription process is slightly more complex, but on the one hand coating-fibre adhesion strength was supposed to be high, and on the other coating was expected to be easier to glue to the housing structure.

Embedded through coating inscribed SOFBGs showed much better characteristics than these yielded by all the previously tested embedding approaches, and none of the previously described issues manifested itself. Interestingly, Bragg peak position for a relaxed structure moved importantly from the original value before embedding. SOFBG1 (T-FBG) moved from $1535.69 \mathrm{~nm}$ before embedding to $1531.73 \mathrm{~nm}$ afterwards (for a relaxed structure), giving the change of $-3.96 \mathrm{~nm}$. SOFGB2 ( $\varepsilon$-FBG) shifted from $1529.39 \mathrm{~nm}$ to $1525.00 \mathrm{~nm}$, yielding the difference of $-4.39 \mathrm{~nm}$. The changes were probably caused by structure shrinkage after cooling down when the printing was finished. The fact that the fibres stayed under compressive stress after printing and did not relax is an argument supporting high fibre-structure adhesion strength. Quantitative test results are presented in Section 4.

\subsection{POFBGs}

The design of the POFBG housing structures is depicted in Figure $2 b$. The difference between the structure 3-D printed of PLA and ABS was the channel depth, which was influenced by the printing nozzle temperature for both materials (around $210{ }^{\circ} \mathrm{C}$ for PLA and $270{ }^{\circ} \mathrm{C}$ for ABS). As POFs are thermoplastic materials, they have to be protected from excessive temperature, not to damage inscribed Bragg gratings. This was achieved by increasing the embedding channel depth. After inserting the fibre to the bottom of the channel, it was covered with a much thicker layer of glue than on embedding SOFBG. The (3-(trimethoxysilyl)propyl)ethylene-diamine elastic glue acted not only as an adhesive, but also a thermoinsulator.

The glue was deposited at the bottom of the channel and along the POF, which provided optimal glue distribution. The fibre was inserted in the channel and moved forth and back along it multiple times in order to assure that it was at the very bottom of the channel and surrounded well with glue. The fibre was mounted with tape on temporary supports at both fibre inlets. The glue was allowed to cure for some time (see below for details). Subsequently, the printing process was resumed, leading to full development of the sensing patch.

In case of the PLA housing, the bottom of the embedding channel was set at the neutral axis of the beam, and its depth was fixed at $750 \mu \mathrm{m}$. It was found to be enough to protect the POFBG from the nozzle temperature $\left(210^{\circ} \mathrm{C}\right)$. The printing bed temperature was set to $60^{\circ} \mathrm{C}$, but the temperature could have been lower at the level of the embedding channel, into which the PLA-POFBG was inserted. These thermal settings did not cause any observable change to the POFBG peak shape. After gluing the grating into the channel and before resuming printing, no grating annealing was observed on the timescale of minutes. The response peak moved from $828.2 \mathrm{~nm}$ before embedding to $826.5 \mathrm{~nm}$ afterwards, which probably came from structure shrinkage after cooling down. Structure shrinkage initially made the peak shape and strength deteriorate, but as the glue was not fully cured at that stage yet, stretching the fibre at both inlets to the structure helped PLA-POFBG regain its initial characteristics. Ultimately, it showed an induced Bragg peak power decrease of $1 \mathrm{~dB}$.

Embedding POFBG in ABS housing was found to be more challenging due to the higher printing nozzle temperature required for $\mathrm{ABS}\left(270^{\circ} \mathrm{C}\right)$. The channel depth was set to $2.3 \mu \mathrm{m}$, which resulted in the bottom of the fibre embedding channel being close to the bottom of the sample and no longer at the neutral axis of the beam. Previous embedding trials showed that $1.15 \mu \mathrm{m}$ deep embedding channel was not deep enough for thermoinsulation from the nozzle. The typical printing bed temperature used for $\mathrm{ABS}\left(105^{\circ} \mathrm{C}\right)$ was found to anneal the fibre on the timescale of minutes. Hence, it was lowered to $60^{\circ} \mathrm{C}$, which prove previously not to cause any adverse effects to POFBG. These printing parameters made the ABS-POFBG withstand the 3-D printing process. The peak shape and strength was unchanged after finishing the printing, but it deteriorated after detaching the structure from the printing bed. This was probably due to structure shrinkage, as this phenomenon is particularly pronounced for ABS - its coefficient of thermal expansion (CTE) is relatively high even as for polymer $\left(73.8 \times 10^{-5} /{ }^{\circ} \mathrm{C}\right)^{11}$. The glue was fully cured at that stage $(1 \mathrm{~h}$ after deposition $)$, so stretching the fibre at the 
inlets to the structure did not help to resolve this issue, as it did for the PLA patch above. Nevertheless, this issue is expected to be easily addressable in this way in the future. The peak shape quality was still good enough for tracking it.

\section{STRAIN RESPONSE OF THE SENSING PATCHES}

Following the embedding process, strain response tests of the sensing patches were performed. The structures were fixed at the parts perpendicular to the embedding channel (see Figure 2) by clamping one of them to an immobilised support, and the other one to a translation stage of $20 \mu \mathrm{m}$ accuracy, so that only the middle beam part of the structure was being strained. For each of the three patches, three straining cycles of gradually increasing and decreasing strain were performed in room temperature. After each strain change, the Bragg peak position was measured at different time intervals: at $\mathrm{t}=0$ $\min , 1 \mathrm{~min}$, and $5 \mathrm{~min}$.

The translation stage used was only able to exert tensile force on the sample, and not compressive one. The first point of all strain plots below in each strain increase cycle is the last stage displacement division at which no change to Bragg peak was visible. The second point in each cycle was the first stage displacement division that caused Bragg peak change. Thus, although the strain plots suggest the first point in each series to be the beginning of strain build-up, in the reality it was somewhere between the first and the second data point. This inaccuracy resulted in a systematic error of up to $0.02 \mathrm{~mm}$, which did not change meaning of the results.

Small but visible length increase from cycle to cycle was noticed for all sensing patches. In order to allow for rough assessment of the length increase, all the strain plots are accompanied by plot showing Bragg wavelength position versus stage displacement. Cycles 2 and 3 of all the patches start with a 2-3 point long period of no stain response, which is not visible on the strain plots.

\subsection{SOFBGs}

During the straining tests of $\varepsilon$-FBG, the sensing patch was interrogated with HBM DI110 running at $1 \mathrm{kHz}$, which allowed for precise tracking of the peak position in time. The time intervals between consecutive straining cycles were about 1 hour. The strain response of the SOFBG sensing patch is shown in Figure 3.

In all cycles a non-linear initial response to strain was observed, which became linear above $0.08 \mathrm{~mm}$ stage displacement. The strain decrease plot in cycle 1 overlapped roughly with the strain increase one, whereas the former was slightly below the latter in cycles 2 and 3.

Strain sensitivity of the linear range of all cycles on strain increase and decrease varied between 0.39 and $0.41 \mathrm{pm} / \mu \varepsilon$. This value is visibly lower than a standard strain sensitivity of silica fibres given in the literature, which is $1 \mathrm{pm} / \mu \varepsilon^{12}$. However, another through coating inscribed SOFBG sensing patch, which was in the preparatory stage, yielded strain sensitivity of $0.95 \mathrm{pm} / \mu \varepsilon$. The grating was embedded in a very similar beam-like structure following almost identical procedure. This strain sensitivity value was very close to the standard silica fibre one. The origin of this difference is unknown to the authors and needs further investigation.

The hysteresis value after cycle 1 of straining was the biggest and of positive magnitude, yielding $401 \mathrm{pm}$. The hysteresis after cycle 2 and 3 was negative and of a decreasing magnitude from cycle to cycle, giving the value of $-222 \mathrm{pm}$ after cycle 2 and $-80 \mathrm{pm}$ after cycle 3 . The response to increasing and decreasing strain in cycle 3 was linear up from $0.06 \mathrm{~mm}$ stage displacement value.

Bragg wavelength changes in time had a relatively low magnitude on the timescale on which they were measured. They were negative on strain increase in all cycles, being the biggest at the highest strain values after $5 \mathrm{~min}$ from strain increment. The highest changes were $-42,-25$ and -18 pm for cycles 1,2 and 3, respectively. On strain decrease the Bragg peak changes in time were mostly positive, being the highest after $5 \mathrm{~min}$ at high strain values. The highest values for cycle 1,2 and 3 were respectively 18, 6 and $9 \mathrm{pm}$. These results show that the fibre tried counteracting the strain changes, trying to compress after tensile strain and to expand after compressive strain.

Note worthily, the structure in cycle 3 showed the smallest strain hysteresis, the highest linearity range and the smallest Bragg wavelength changes in time. These suggest that longer term operation of the structure can yield favourable characteristics from the viewpoint of sensor applications. 

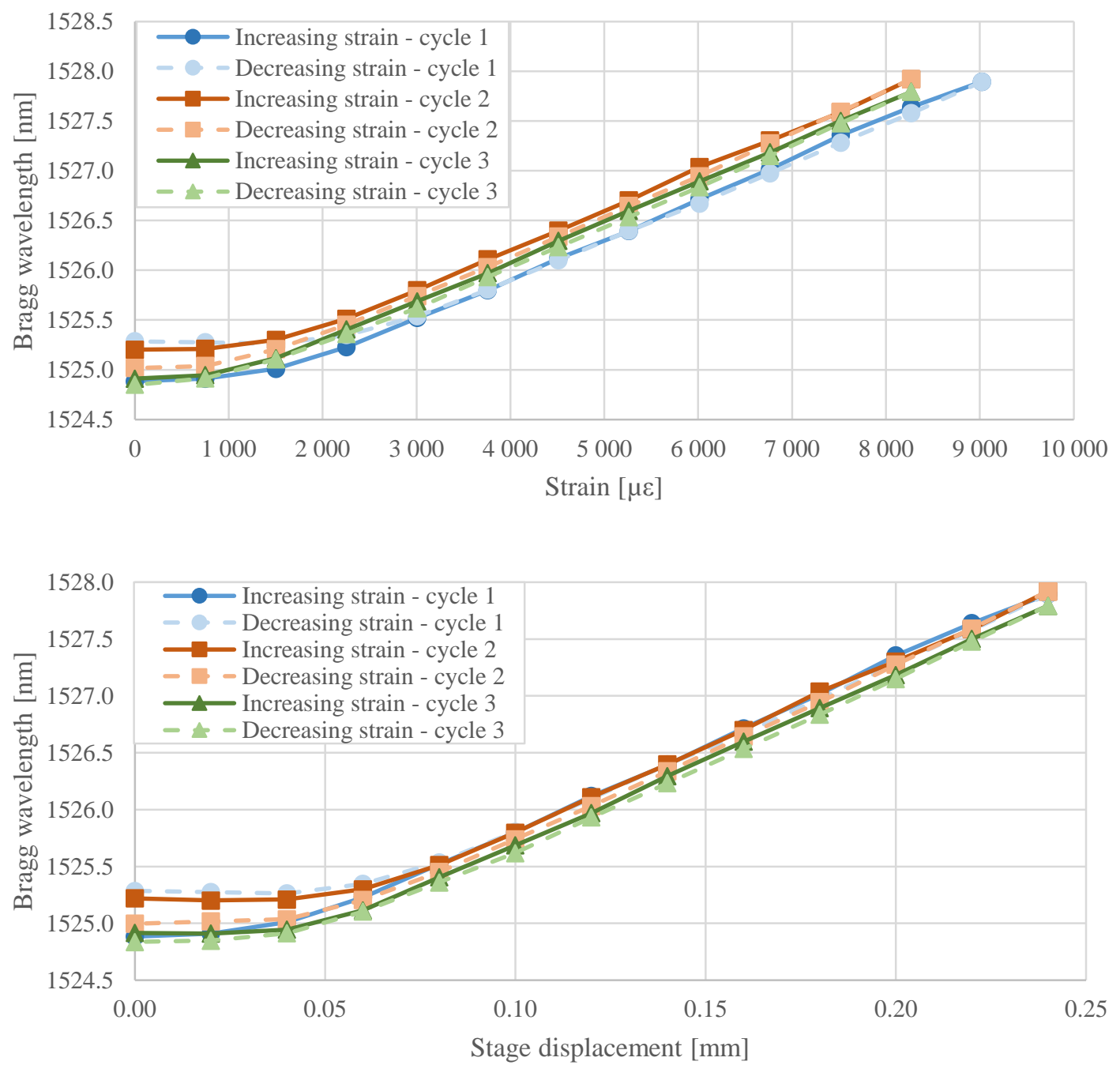

Figure 3. $\varepsilon$-FBG (SOFBG) Bragg reflection peak immediately after strain change in the function of strain and testing stage displacement for the sensing patch of ABS.

\subsection{POFBG embedded in the PLA sensing patch}

The sample was interrogated in the same way as for POFGB inscription (see Figure 1b). The peak position measurements were taken with OSA and not with an interrogator, so they could not have been recorded exactly immediately strain change as it was for embedded SOFBGs. Time intervals between consecutive straining cycles were over 15 hours for the cycle 12 interval, and around 2 hours for the cycle 2-3 one. The straining response of POFBG embedded in PLA was plotted in Figure 4.

The strain increase in cycle 1 showed the lowest linearity of all. This might be attributed to the POF taking optimal position within the housing, compensating for any possible slack that remained from the embedding stage. On strain decrease, a response of good linearity was observed down to $0.10 \mathrm{~mm}$ stage displacement. Structure length increased from cycle to cycle, because the increase in strain in cycles 2 and 3 started at higher values of stage displacement. What is more, at lower stage displacement values a non-linear strain increase was observed in cycle 2 and 3, as opposed to the fibre behaviour in cycle 1 . This also contradicted some literature results for mounted fibres ${ }^{13}$. 

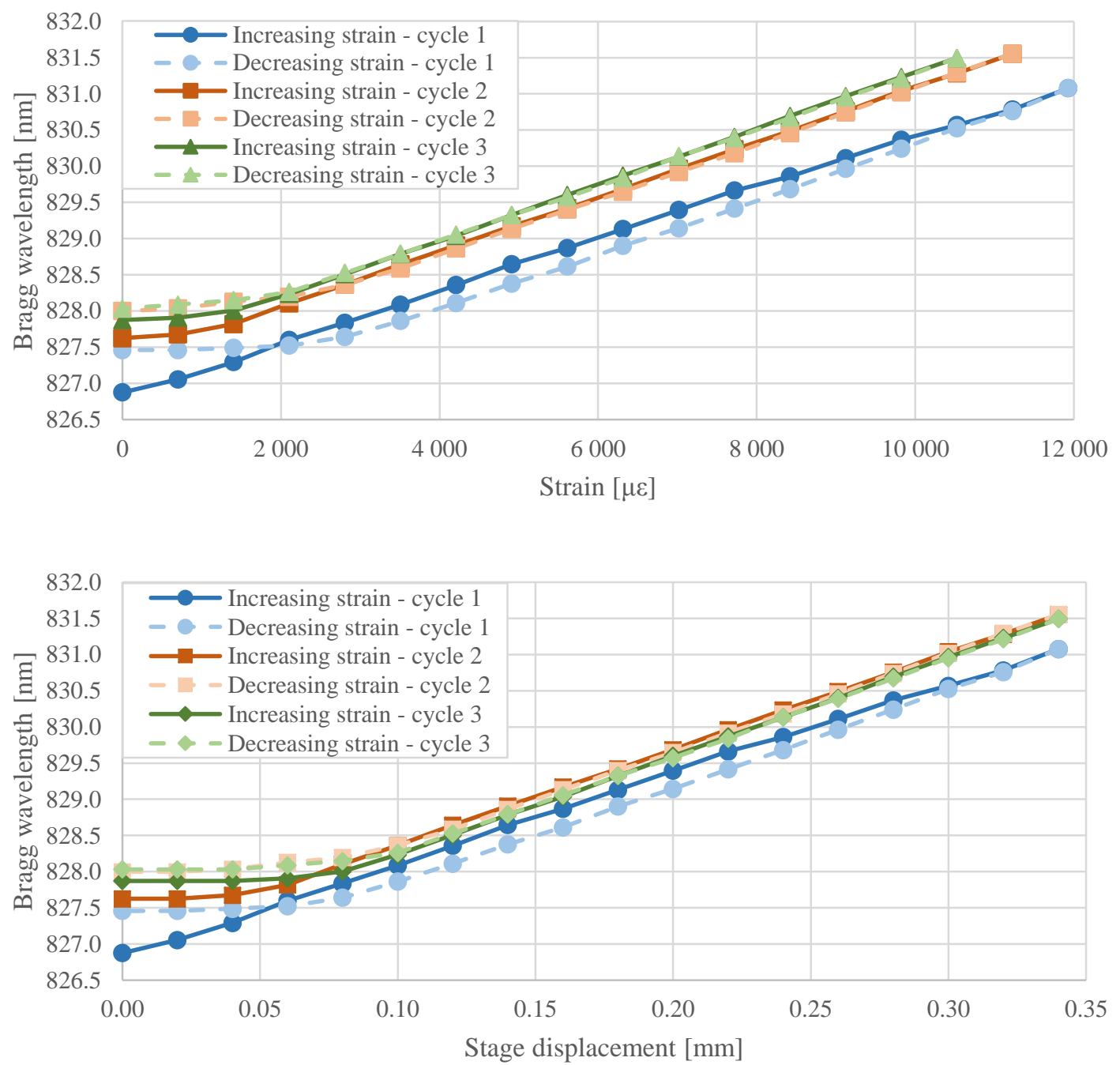

Figure 4. PLA-POFBG Bragg reflection peak immediately after strain change in the function of strain and testing stage displacement for the sensing patch of PLA.

Apart from cycle 1, strain increase and decrease almost exactly overlapped, showing good repeatability of strain response within one cycle. Moreover, in the linear range of cycles 2 and 3 the slope of the increase and decrease of strain showed almost the same value. This confirmed repeatability of strain sensitivity, which is important for sensing applications.

Strain sensitivity, calculated based on the linear strain response ranges (excluding strain increase in cycle 1), yielded the value of $0.38 \mathrm{pm} / \mu \varepsilon$. It was more than a half of strain sensitivity of this fibre itself, reported to be in the range of $0.54-0.77$ $\mathrm{pm} / \mu \varepsilon^{14}$. A speculative explanation of that phenomenon may be that part of the stress was shielded by the glue, but a more definite conclusion would require more investigation.

The hysteresis of strain response was always positive, and its value decreased with increasing cycle, yielding $580 \mathrm{pm}, 380$ $\mathrm{pm}$ and $160 \mathrm{pm}$ respectively in cycle 1,2 and 3. Bragg wavelength changes with time were relatively small and rather irregular, being the biggest for measurements taken $5 \mathrm{~min}$ after strain change. The maximal positive and negative values for cycles 1, 2 and 3 were respectively $22 /-47 \mathrm{pm}, 1 /-58 \mathrm{pm}$ and 0/-44 pm.

\subsection{POFBG embedded in the ABS sensing patch}

The straining characteristics of this sensing patch were taken keeping the same experimental conditions as for the PLA sample above (see the first paragraph of Section 4.2). The results are shown in Figure 5. 
There was a notable difference between the absolute Bragg peak positions in cycle 1 and $2 / 3$ of some $340 \mathrm{pm}$. This may have been due to longer time elapsed between cycle 1 and 2 than 2 and 3, but the same experimental conditions for the PLA patch did not yield similar results. Consecutive straining cycles show ever lower absolute Bragg wavelength values.

There was a clear offset between strain increase and decrease data series in cycle 1 as compared to cycle 2 and 3 , although the latter also did not overlap very closely in comparison to the PLA sensing patch (see Figure 4). This difference in cycle 1 can be perhaps explained by the fibre finding optimal position within the housing structure. Linear strain response manifested itself above $0.06 \mathrm{~mm}$ stage displacement value. For lower stage displacements, all straining cycles but strain increase in cycle 1 showed clear strain sensitivity dip.

The strain sensitivity in the linear range was calculated to be about $0.14-0.15 \mathrm{pm} / \mu \varepsilon$, being some 4.5 time lower than the values reported for this fibre itself, which fall between 0.54-0.77 $\mathrm{pm} / \mu \varepsilon^{14}$. As in the case of the PLA sensing patch, a hypothetical reason might be the elastic glue shielding part of the strain, but such an explanation can by no means be taken for definite without further investigation.
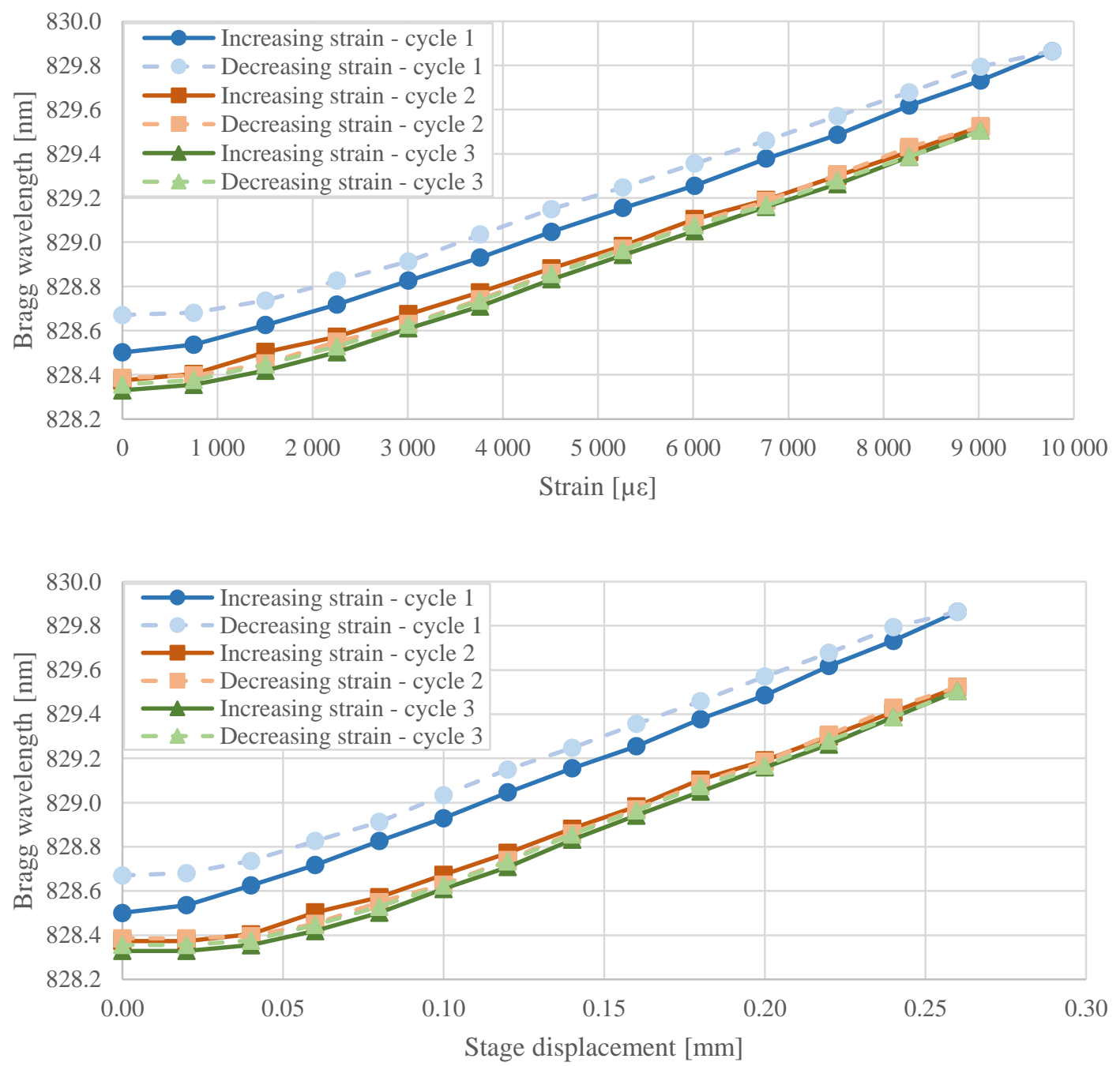

Figure 5. ABS-POFBG Bragg reflection peak immediately after strain change in the function of strain and testing stage displacement for the sensing patch of ABS.

The strain hysteresis was always positive and the biggest in the first strain cycle $(169 \mathrm{pm})$. The other cycles yielded much smaller hysteresis values (12 pm in cycle 2 and $27 \mathrm{pm}$ in cycle 3). Bragg wavelength changes in time were very small in 
all cycles. The maximal positive and negative values for cycles 1, 2 and 3 measured after 5 min strain change were respectively 13/-9 pm, 4/-11 pm and 5/-11 pm.

\subsection{Comparison of the strain response of the embedded FBGs}

Summary of straining characteristics has been collated in Table 1.

All sensing patches showed two regions of different strain response. Up to the threshold of $0.06-0.08 \mathrm{~mm}$ stage displacement the response to strain was non-linear, of a gradually increasing sensitivity. Above the threshold the strain characteristic was linear.

In case of sensing patches containing POFBGs, the strain increase in cycle 1 was very different from all others. It can perhaps be linked with fibre finding optimal position within structure after 3-D printing. This effect was not observed for the SOFBG patch.

The strain sensitivity values of all sensing patches caused some confusion. Visibly different values for two SOFBG patches were found for almost identical design and embedding procedure. As to POFBGs, the strain sensitivity values for PLA and ABS patches were very different from each other and from the value reported in the literature for unembedded fibre. This clearly shows the need for research into understanding the mechanism determining the sensitivity values.

Although the strain sensitivity changed from sample to sample, its values were all very repeatable over 3 straining cycles. The maximal shifts of Bragg wavelength in time after strain change on the timescale of 5 minutes was -58 pm, and was normally much lower. These are all favourable characteristics from the point of view of applications in sensing.

For all patches an increase in structure length after straining cycle was observed. Its maximal value was around 0.04 mm for SOFBG and PLA-POFBG sensing patches. It is speculated that all the structures, having been made of elastic materials and not having exceeded their strain elastic limits, would at least partly compensate for the length increase on the longer time scale. Nonetheless, 3-D printed structures are more complex than bulk material, and definite conclusions would require more investigation, which is beyond the scope of this paper.

Comparison of POFBGs embedded in PLA and ABS showed that the latter had favourable characteristics from the viewpoint of sensing applications. The strain hysteresis in cycle 1 was $\sim 3.5$ times smaller in the ABS-POFBG patch, and almost 6 times smaller in cycle 3. The biggest maximal value of Bragg peak shift after 5 min of strain change was 4.5 times smaller for the ABS-POFBG patch.

Table 1. Summary of straining characteristics for the manufactured sensing patches.

\begin{tabular}{|l|c|c|c|}
\hline & SOFBG & PLA-POFBG & ABS-POFBG \\
\hline $\begin{array}{l}\text { Measured strain sensitivity } \\
\text { (literature value for unembedded fibre) }[\mathrm{pm} / \mu \varepsilon]\end{array}$ & $\begin{array}{c}0.39-0.41 \text { or } 0.95 \\
(\sim 1.0)^{12}\end{array}$ & $\begin{array}{c}0.38 \\
(0.54-0.77)^{14}\end{array}$ & $\begin{array}{c}0.14-0.15 \\
(0.54-0.77)^{14}\end{array}$ \\
\hline Hysteresis after cycle 1; cycle 2; cycle 3 [pm] & $401 ;-222 ;-80$ & $580 ; 380 ; 160$ & $169 ; 12 ; 27$ \\
\hline $\begin{array}{l}\text { Maximal positive Bragg peak change after 5 min from } \\
\text { strain change in cycle 1; cycle 2; cycle 3 [pm] }\end{array}$ & $18 ; 6 ; 9$ & $22 ; 1 ; 0$ & $13 ; 4 ; 5$ \\
\hline $\begin{array}{l}\text { Maximal negative Bragg peak change after 5 min from } \\
\text { strain change in cycle 1; cycle 2; cycle 3 [pm] }\end{array}$ & $-42 ;-25 ;-18$ & $-47 ;-58 ;-44$ & $-9 ;-11 ;-11$ \\
\hline
\end{tabular}

\section{TEMPERATURE RESPONSE OF THE SENSING PATCHES}

After strain tests, temperature response of the samples was assessed. They were placed on the hotplate under a specially prepared thermoinsulating cover. It had an opening for a mercury thermometer tip, which was used to control the temperature. The maximum temperature during the test was $\sim 43{ }^{\circ} \mathrm{C}$, as this is enough for most medical applications and bio-applications.

Although thermal uniformity of the hotplate was found to be better than this of an oven, it was not complete. The issue was especially visible with the SOFBG patch, as its observed temperature sensitivity was very high. Cycles of heating up 
and cooling down were clearly visible at lower temperatures with the SOFBG patch. Hence, it has to be borne in mind that the non-linear temperature response of the SOFBG patch was at least partly influenced by the testing set-up.

\subsection{SOFBGs}

The SOFBG patch was interrogated on the HBM DI110. Although T-FBG was meant to measure the temperature, due to the testing set-up $\varepsilon$-FBG was found to give a more credible reading. It was placed centrally in the sensing patch, and hence was closer to the central heating region of the hotplate. Because of that, when the central $\varepsilon$-FBG showed the temperature to stabilise or start decreasing, the temperature reading at T-FBG was still in increase.

On increasing temperature series the highest readings for each temperature setting were noted, whereas the lowest temperature readings were taken on temperature decrease. The resulting plot is shown in Figure 6. It shows roughly linear response to temperature. The non-linearities are believed to be caused at least partly by the testing set-up.

Temperature sensitivity for increasing strain equalled to $146 \mathrm{pm} /{ }^{\circ} \mathrm{C}$, and for decreasing strain $169 \mathrm{pm} /{ }^{\circ} \mathrm{C}$. These values were 15-17 times higher than temperature sensitivity of unembedded silica grating, which is around $10 \mathrm{pm} /{ }^{\circ} \mathrm{C}^{12}$. Such an increase in temperature sensitivity comes probably from the fact that the linear CTE of ABS $\left(73.8 \times 10^{-6} /{ }^{\circ} \mathrm{C}^{11}\right)$ is over 130 times higher that this of fused silica $\left(0.55 \times 10^{-6} /{ }^{\circ} \mathrm{C}^{11}\right)$. As fibre-structure adhesion is high, the fibre is forced to expand with the structure on heating up, which effectively makes it strain. However, temperature sensitivity of silica FBGs is mostly contributed to by thermo-optic coefficient of silica and not its CTE, which is the reason why 130 increase of CTE should not be expected to give an equivalent increase in temperature sensitivity of the embedded grating.

The temperature cycling hysteresis of the temperature response of $-526 \mathrm{pm}$ was observed. As it was measured at room temperature, the aforementioned limitations of the testing set-up should not have influenced it. The origin of the thermal hysteresis remains unknown to the authors.

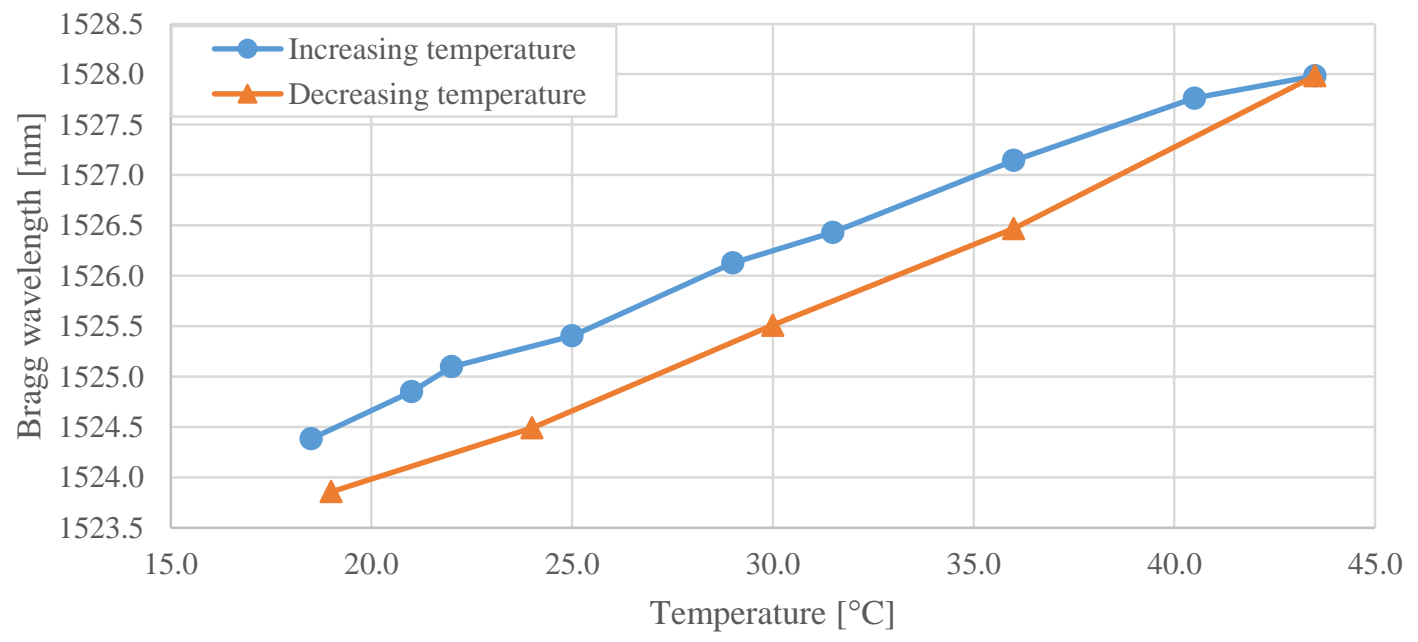

Figure 6. $\varepsilon$-FBG (SOFBG) Bragg reflection peak immediately after reaching given temperature in the function of temperature value for the sensing patch of ABS.

\subsection{POFBGs}

The temperature response of both POFBG sensing patches was very unstable. The Bragg peak was visibly decreasing with time at the temperatures of $30-35^{\circ} \mathrm{C}$, which is at least $15^{\circ} \mathrm{C}$ below the annealing temperatures for unembedded POFBGs noticed by the authors. Perhaps the phenomenon had to do with annealing of structure material rather than the grating itself, but a definite answer to that could not be formulated. Only the PLA-POFBG structure was managed to be partly thermally stabilised after 3 hours annealing at $55^{\circ} \mathrm{C}$ (more than $10^{\circ} \mathrm{C}$ above maximal intended temperature of operation). At the end of the annealing the Bragg wavelength had decreased by $13.92 \mathrm{~nm}$ to $814.08 \mathrm{~nm}$. The thermal response of this grating was plotted in Figure 7.

Thermal hysteresis after cycling was $-220 \mathrm{pm}$, which prove that the thermal stabilisation was not complete. The resulting temperature sensitivity was $30-40 \mathrm{pm} /{ }^{\circ} \mathrm{C}$, whereas usually reported values are of similar magnitude but negative ${ }^{3}$. 
Nevertheless, is has to be noted that the temperature response of POF is often fibre-dependent, and strongly depends on humidity ${ }^{3}$.

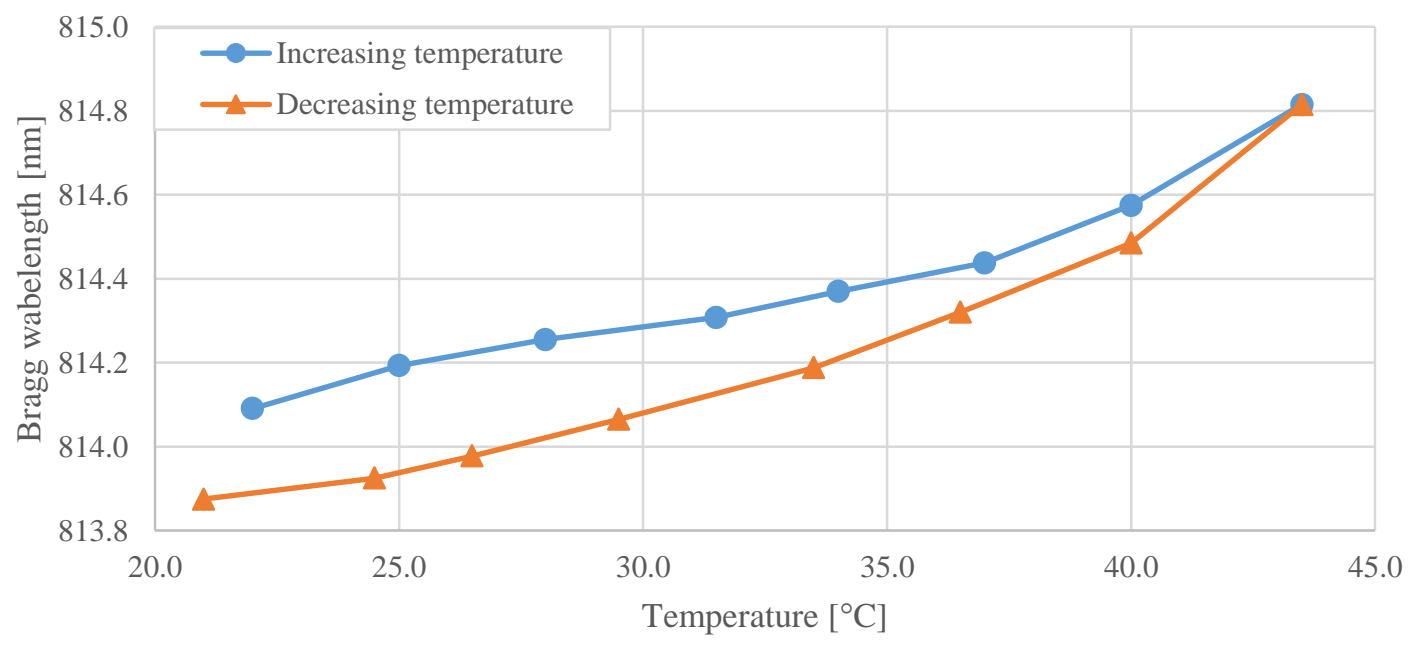

Figure 7. PLA-POFBG Bragg reflection peak immediately after reaching given temperature in the function of temperature value for the sensing patch of PLA.

\section{CONCLUSIONS}

In this paper an embedded SOFBG in an FDM 3-D printed housing structure yielding a double grating temperaturecompensated strain sensor is reported. We also report the first ever integration of POFBG with a 3-D printed sensing patch for strain or temperature sensing. The embedding process is expected to facilitate rapid, flexible and easily-customisable optical fibre sensor production in the future.

Different strain sensitivities of the sensing patches were reported: 0.40 and $0.95 \mathrm{pm} / \mu \varepsilon$ for SOFBG embedded in ABS, $0.38 \mathrm{pm} / \mu \varepsilon$ for POFBG in PLA and $0.15 \mathrm{pm} / \mu \varepsilon$ for POFBG in ABS. Strain sensitivity was repeatable and strain response did not change much in time on the timescale of minutes. Strain hysteresis was found to decrease with straining cycles, the smallest value of 12 pm having been measured for the ABS-POFBG patch.

Compared to PLA, ABS housing material has shown favourable characteristics from the viewpoint of sensing applications. The strain hysteresis was almost 6 times smaller in cycle 3 for ABS-POFBG. The biggest maximal value of Bragg peak shift after 5 min of strain change was 4.5 times smaller than for the PLA-POFBG patch.

Temperature sensitivity of the SOFBG sensing patch was found to be $146-169 \mathrm{pm} /{ }^{\circ} \mathrm{C}$, which was up to 17 times higher than the value for an unembedded silica grating. The temperature response of POFBG sensing patches was unstable, yielding $30-40 \mathrm{pm} /{ }^{\circ} \mathrm{C}$ for PLA-POFBG.

The authors will be undertaking further research into factors influencing strain sensitivity and the ways of thermally stabilising 3-D printed sensing patches.

\section{ACKNOWLEDGEMENT}

The research leading to these results has received funding from the People Programme (Marie Curie Actions) of the European Union's Seventh Framework Programme FP7/2007-2013/ under REA grant agreement nº 608382. 


\section{REFERENCES}

[1] S. H. Huang, P. Liu, A. Mokasdar et al., "Additive manufacturing and its societal impact: A literature review," International Journal of Advanced Manufacturing Technology, 67(5-8), 1191-1203 (2013).

[2] T. J. Horn, and O. L. A. Harrysson, "Overview of current additive manufacturing technologies and selected applications," Science Progress, 95(3), 255-282 (2012).

[3] D. J. Webb, [Polymer Fibre Bragg Grating Sensors and their Applications] CRC Press, United Kingdom(2015).

[4] C. Broadway, D. Gallego, G. Woyessa et al., "Polymer optical fibre sensors for endoscopic optoacoustic imaging," Proc. SPIE. 9539(2015).

[5] C. Broadway, D. Gallego, G. Woyessa et al., "Fabry-Perot micro-structured polymer optical fibre sensors for opto-acoustic endoscopy," Proc. SPIE. 9531(2015).

[6] C. A. F. Marquess, A. Pospori, D. Sáez-Rodríguez et al., "Fiber-optic liquid level monitoring system using microstructured polymer fiber Bragg grating array sensors: Performance analysis," Proc. SPIE. 9634.

[7] R. R. J. Maier, W. N. MacPherson, J. S. Barton et al., "Embedded fiber optic sensors within additive layer manufactured components," IEEE Sensors Journal, 13(3), 969-979 (2013).

[8] A. Kantaros, and D. Karalekas, "Fiber Bragg grating based investigation of residual strains in ABS parts fabricated by fused deposition modeling process," Materials and Design, 50, 44-50 (2013).

[9] H. Dobb, D. J. Webb, K. Kalli et al., "Continuous wave ultraviolet light-induced fiber Bragg gratings in few- and single-mode microstructured polymer optical fibers," Optics letters, 30(24), 3296-8 (2005).

[10] D. Saez-Rodriguez, K. Nielsen, H. K. Rasmussen et al., "Highly photosensitive polymethyl methacrylate microstructured polymer optical fiber with doped core," Optics Letters, 38(19), 3769--3772 (2013).

[11] W. E. Forsythe, [Smithsonian Physical Tables (9th Revised Edition)] Knovel.

[12] A. Cusano, A. Cutolo, and J. Albert, [Fiber Bragg grating sensors: recent advancements, industrial applications and market exploitation] Bentham Science Publishers Ltd., Saif Zone, Sharjah, United Arab Emirates(2011).

[13] A. Abang, and D. J. Webb, "Influence of mounting on the hysteresis of polymer fiber Bragg grating strain sensors," Optics Letters, 38(9), 1376-1378 (2013).

[14] A. Pospori, C. A. F. Marques, M. G. Zubel et al., "Annealing effects on strain and stress sensitivity of polymer optical fibre based sensors " Proc. SPIE. 9886(2016). 